Www.jmscr.igmpublication.org

Index Copernicus Value: 79.54

ISSN (e)-2347-176x ISSN (p) 2455-0450

crossref DOI: https://dx.doi.org/10.18535/jmscr/v7i4.126

Original Article

\title{
Bone Marrow Spectrum of Acute Leukaemia's at a Tertiary Care Hospital- An Experience
}

\author{
Authors \\ Manzoor Ahmad Sofi ${ }^{1}$, Shaheena Parveen ${ }^{1}$, Shareefa Akhter ${ }^{2}$, Nausrat Ali \\ ${ }^{1}$ Senior Resident Department of Pathology, GMC, Srinagar, J\&K, India \\ ${ }^{2}$ Senior Resident, Department of Hematopathology, SKIMS, SOURA, Srinagar, J\&K, India \\ Email: akhtersharefa@gmail.com \\ *Corresponding Author \\ Dr Nusrat Bashir
}

Assistant Professor, Department of Pathology, GMC, Srinagar, J\&K, India 190010

Contact no: 9419029270

\begin{abstract}
Background: The diagnosis of acute leukaemia's requires a multifaceted approach.

Aims: The present study was undertaken to highlight the bone marrow spectrum of acute leukaemia's in our settings.

Materials and Methods: The present study was undertaken during the period of January 2016 to December 2018 at Hematology unit, Department of Pathology, Government Medical College, Srinagar, Kashmir. Bone marrow aspiration was done in all newly suspected cases of acute leukaemia's.

Results: During the study period, 45 cases were reported as acute leukaemia. The age distribution ranged from 7 to 85 years. Out of total 45 cases 37 had as acute myeloid leukaemia (AML) and 8 cases were of acute lymphoblastic leukaemia (ALL).

Conclusion: Diagnosis of acute leukaemia's is a multi parametric approach $i$ combining morphology, cytochemistry, immunophenotyping and cytogenetics.

Keywords: Acute leukaemia, Bone marrow, Myeloid, Lymphoid.
\end{abstract}

\section{Introduction}

Acute leukemias are characterized by neoplastic proliferation of hematopoietic stem cells and are broadly classified into two main groups namely: Acute Myeloid Leukemia (AML) and Acute Lymphoid Leukemia (ALL) based on the cellular presentation of primary stem cell defect ${ }^{[1]}$. World Health Organization (WHO) further subclassifies them based on morphology, cytochemistry, immunophenotyping, cytogenetic and molecular genetics studies $^{[2]}$. The latest WHO classification of the acute leukemias differs from the FAB classification in that greater than or equal to $20 \%$ blasts are used for diagnosis of acute leukemias ${ }^{[3]}$. Acute myeloid leukemia is a complex disease with considerable phenotypic and genotypic heterogeneity. There are more than 100 recurring cytogenetic abnormalities observed in AML and 
numerous point mutations. In addition, patients with the clonal, recurring cytogenetic abnormalities $\mathrm{t}(8 ; 21)(\mathrm{q} 22 ; \mathrm{q} 22), \operatorname{inv}(16)(\mathrm{p} 13 \mathrm{q} 22)$ or $\mathrm{t}(16 ; 16)(\mathrm{p} 13 ; \mathrm{q} 22)$, and $\mathrm{t}(15 ; 17)(\mathrm{q} 22 ; \mathrm{q} 12)$ should be considered to have AML regardless of the blast percentage ${ }^{[4]}$. By assessing morphologic features together, a majority of cases of AML and ALL can be accurately diagnosed. In some cases of poorly differentiated acute leukemia, however, the morphologic features may be equivocal, requiring additional studies. The diagnosis of BALL is established by immunophenotyping, commonly by flow cytometry (FC), which shows immature B lineage. Many cases of B-ALL harbor recurrent chromosomal abnormalities, including balanced chromosomal translocations, which are often critical determinants of prognosis ${ }^{[5]}$.

\section{Materials and Methods}

The present study was undertaken during the period of January2016 to December 2018 at Hematology unit, Department of Pathology, Government Medical College, Srinagar, Kashmir. Case selection was based on clinical features and supported by laboratory evidences. Bone marrow aspiration was subsequently carried out after obtaining written consent from the patient or the guardian. All new cases of acute leukaemia were included in our study, however, treated cases of leukemia and those with blast crisis in chronic myeloid leukemia were excluded. Complete haemogram was done. The peripheral and bone marrow aspiration smears were stained by Leishman stain for all cases and examined in detail. In all cases, the following cyto-chemical stains were employed for diagnosis and subtyping of leukemias.

1. MPO-Myelo-peroxidase stain

2. PAS-Per-iodic Acid Schiff Stain

\section{Results}

During the study period, 45 cases were reported as acute leukemia. The age distribution ranged from 7 to 85 years (Table-1). Out of 45 patients, 28 were male patients and 17 were female patients.
Males were slightly affected more by the disease when compared to females.

Most patients with AML presented with anemia (median hemoglobin $8 \mathrm{~g} \%$ ), thrombocytopenia (median platelet count 40,000-50,000/ $\mathrm{LL}$ ), and leukocytosis (median white blood cell count 10,000-20,000/ $\mu \mathrm{L})$. Most common clinical presentation was generalised fatigue followed by fever and lymphadenopathy. Careful examination detected blasts in peripheral smears in most patient with one of the patient showing marked pancytopenia with no blasts in the peripheral smear, however, proved to be a case of aleukemic leukemia on bone marrow examination.

Bone marrow aspiration was performed in all the 45 patients. Cases were further subtyped using the FAB criteria on morphological and cytochemical grounds into different subtypes M0 to M7 and L1 to L3(Table-2). The demonstration of myeloid lineage was done on the basis of morphology and by positive myeloperoxidase stain. These stains were uniformly negative in ALL cases which showed in turn, positivity for PAS stain. The monocytic lineage cells were demonstrated based on morphological grounds.

The distribution of Acute Leukemia patients into various subtypes were; (Table-3)

AML M0 in 6 patients (13.3\%)

AML M1 in 6 patients (13.3\%)

AML M2 in 9 patients (20\%)

AML M3 in 9 patients (20\%)

AML M4/5 in 1 patients (2.2\%)

AML M5 in 1 patients $(2.2 \%)$

AML M6 in 1 patient $(2.2 \%)$

AML with MDS in 4 patients $(8.8 \%)$

ALL in 8 patients $(17.7 \%)$

No patients of M7 were encountered in this study [Table2].

\section{Acute Myeloblastic Leukemia with Minimal Maturation (AML-M0)}

The diagnosis of AML-M0 (Fig-1) was made in 6 patients constituting $13.3 \%$ of all acute leukemia patients. The age ranged from 60-75 years There were five male patients and one female patient. In all the cases, myeloblast constituted more than 
$90 \%$ of the cells with a mean blast count of $95 \%$. Cytochemical stains showed MPO negative blasts. We reported these cases on morphology as MPO negative acute leukemia. On immunophenotyping these blasts were of myeloid lineage with positive CD117 and negative lymphoid markers.

\section{Acute Myeloblastic Leukemia without Maturation (AML-M1)}

The diagnosis of AML-M1 was made in 6 patients constituting $13.3 \%$ of all patients. The age ranges 60-85 years. There were five male patients and one female patient. In all the cases, myeloblast constituted $92 \%$ of all cells counted. The maturing component accounted for less than $10 \%$ of the cells. Cytochemical stains showed MPO positivity in more than $3 \%$ of the blasts.

\section{Acute Myeloblastic Leukemia with Differentiation (AML-M2)}

AML-M2 comprised of 9 patients constituting $20 \%$ of all the patients. The age ranged from 1975 years. There were 5 males and 4 females. Bone marrow smears were hypercellular. Blast percentage was $62 \%$. Auer rods were seen in most of the patients. MPO was strongly positive all cases.

\section{Acute Promyelocytic Leukemia (AML-M3)}

Nine patients were diagnosed as AML-M3(Fig2)comprising $20 \%$. The age ranged from $20-70$ years. There were six male and three female patients. Bone marrow examination revealed M:E ratio of 16:1. The predominant cells were leukemic promyelocytes constituting 90\%. Many cases showed multiple auer rods (Faggots). Cytochemical stains for MPO revealed strong positivity. Out of 9 cases 5 were hypergranular APML cases and 4 had hypogranular APML

\section{Acute Myelomonocytic Leukemia (AML-M4/5)}

The diagnosis of AML-M4/5 was made in 1 patient. This patient was a 20 year old female.

Bone marrow aspiration was done in the patients. The average blast count was $75 \%$, which included cells of both myeloid series (20\%) and monocytoid series (20\%). Cytochemical stains showed positive MPO reactivity in the myeloid series whereas the monocytoid series e
Acute Monocytic Leukemia (AML-M5)

There was one 48 year old female who was diagnosed as AML-M5. Bone marrow aspiration showed a hypercellular marrow with predominance of monoblasts (> 80\%). MPO showed negative staining and PAS stains were also negative.

Acute erythroid leukemia (AML-M6)

A 46 year old male patient presented with pancytopenia and nrbcs in peripheral smear. Bone marrow examination revealed bizarre megaloblastic and often multinucleated erythroid precursors. Karyorrhexis was seen in some cells. There were $>20 \%$ blasts among nonerythroid cells with $>50 \%$ of the marrow nucleated elements being erythroid.

Acute leukemia with myelodysplastic changes (AML with MDS)

Three patients, two males and one female were diagnosed as AML with myelodysplasia related changes. They were all elderly patients between 70- 85 years. All cases presented with pancytopenia and subleukemic picture. Bone marrow examination revealed more than 50\% dysplastic cells in at least two cell lineages with > $20 \%$ blasts in nucleated cells counted.

\section{Acute lymphoblastic leukemia (ALL)}

All ALL (Fig-3) patients in our set up were of paediatric age group with most common presentation as Fever.We received only bone marrow aspirate smears from paediatric hospital .All cases had hypercellular marrow almost replaced by lymphoid blasts.

Immunophenotyping and cytogenetics were done from outside laboratory as the facility is not available in our hospital.

\section{Table 1}

\begin{tabular}{|l|c|c|}
\hline & Males & Females \\
\hline $1-10$ & 0 & 2 \\
\hline $11-20$ & 4 & 4 \\
\hline $21-30$ & 3 & 2 \\
\hline $31-40$ & 2 & 1 \\
\hline $41-50$ & 3 & 4 \\
\hline $51-60$ & 4 & 2 \\
\hline $61-70$ & 7 & 0 \\
\hline $71-80$ & 5 & 0 \\
\hline $81-90$ & 0 & 2 \\
\hline Total & $\mathbf{2 8}(\mathbf{6 2 . 2 \%})$ & $\mathbf{1 7}(\mathbf{3 7 . 7 \% )}$ \\
\hline
\end{tabular}


Table 2

\begin{tabular}{|l|c|}
\hline & \\
\hline AML & 33 \\
\hline AML with MDS & 3 \\
\hline Aleukemic leukemia & 1 \\
\hline ALL & 8 \\
\hline Total & 45 \\
\hline
\end{tabular}

Table 3

\begin{tabular}{|l|c|c|c|}
\hline & Male & Female & Total \\
\hline AML-M0 & 5 & 1 & $6(13.3 \%)$ \\
\hline AML-M1 & 5 & 1 & $6(13.3 \%)$ \\
\hline AML-M2 & 6 & 3 & $9(20 \%)$ \\
\hline AML-M3 & 6 & 3 & $9(20 \%)$ \\
\hline AML-M4/5 & 0 & 1 & $1(2.2 \%)$ \\
\hline AML-M5 & 0 & 1 & $1(2.2 \%)$ \\
\hline AML-M6 & 1 & 0 & $1(2.2 \%)$ \\
\hline AML with MDS & 2 & 1 & $3(6.6 \%)$ \\
\hline ALL & 2 & 6 & $8(17.7 \%)$ \\
\hline Aleukemic leukemia & 1 & 0 & $1(2.2 \%)$ \\
\hline Total & $\mathbf{2 8}$ & $\mathbf{1 7}$ & $\mathbf{4 5}$ \\
\hline
\end{tabular}

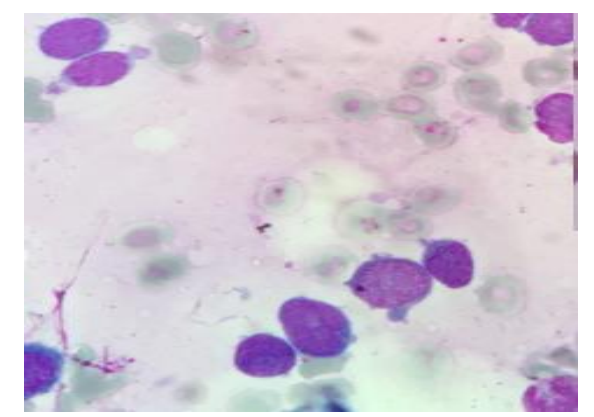

Fig-1: Bone marrow in AML- with minimal differentiation.

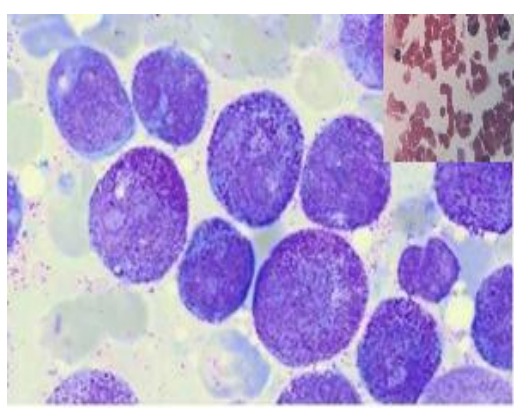

Figure- 2: Bone marrow aspirate in APML showing hypergranular promyelocytes with inset showing MPO positivity.

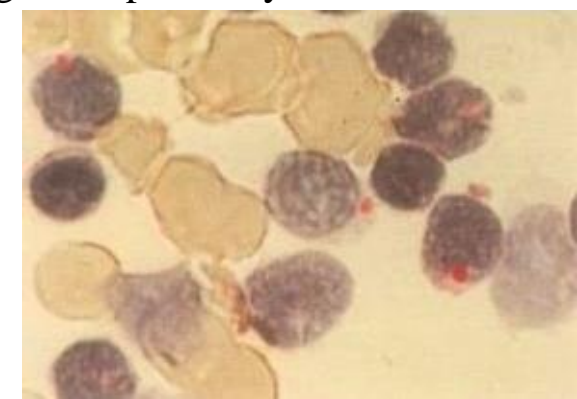

Figure-3: Bone marrow showing block positive lymphoid blasts in ALL.

\section{Discussion}

All acute leukemias are required to be classified either as AML or ALL. This is crucial for two interdependent reasons: one, to choose the most appropriate ancillary investigation for exact subtyping and second to offer the most appropriate therapy. The advent of targeted gene therapy has made it imperative for this subtyping to be done ${ }^{[6]}$. In this study of ours, 45 patients were diagnosed as acute leukemia after being subjected to detailed clinical history and examination followed by a complete hematological work up along with special stains and bone marrow examination. There were 33 patients diagnosed as AML, 3 patients as AML with MDS and 8 patients with ALL. While our study showed only a slight male preponderance (M: $\mathrm{F}=1.64: 1$ ), a higher occurrence in men was noted in D' Costa et al. and Anuradha Kusum et al.'s studies i.e. 2.7:1 and 2.3:1, respectively. ${ }^{[7,8]}$

The main presenting symptoms of our AML patients were fever and generalized weakness. Similar presenting symptoms were observed by studies conducted by Advani et al., ${ }^{[9]}$, Shome et al., ${ }^{[10]}$ and Mathur et al., ${ }^{[1]}$. As in concordance with the study of Mathur et al, pallor was found in $100 \%$ of the patients.

Our study showed an incidence of Lymphadenopathy $(3.5 \%)$ which correlates with the study conducted by Advani et al. (4\%), whereas in other studies done by Shome et al and Mathur et al lymphadenopathy was seen in more than $30 \%$ of patients.

Our study showed that a definitive subtyping of acute leukemia as AML or ALL, purely by morphology, could be made in only 20 of the cases. We used only 2 cytochemical markers (namely myeloperoxidase and periodic acid Schiff) in the cases wherever cytochemistry was performed. The use of these two cytochemical markers helped in the typing of 10 more cases of acute leukemia. Thus, of the 45 cases of acute leukemia, 30 cases could be subtyped as AML or ALL on morphology and cytochemistry. The acute leukemia cases were diagnosed as acute 
myeloid leukemia on the basis of morphology, cytochemistry and diagnostic Auer rods which are crystalline structures seen only in AML or in high grade myelodysplastic syndrome. They are never seen in lymphoblasts ${ }^{[12]}$. All the 8 cases of ALL were diagnosed on morphology and cytochemistry where the PAS reaction showed block positivity. The separation of the various entities belonging to the group of myeloid neoplasms is best achieved by a multiparametric approach. Loffler $\mathrm{H}$, et al. opined that ALL diagnosed by cytochemistry must be confirmed by immunophenotyping in all the cases. Immunophenotyping of the leukemic blasts is essential for the diagnosis and confirmation of acute leukemia into one of the two types (AML or ALL). The detection system can either be on flow cytometry or immunohistochemistry ${ }^{[13]}$. However, the importance of bone marrow morphology cannot be ignored in reaching a correct diagnosis. Additionally, it might be the only approach available in many countries and the one upon which the practicing hematologist has to rely. Morphology supplemented by immunohistochemistry with markers reactive in routinely processed tissues represents a powerful diagnostic tool on its own. Its results can be usually easily correlated to those obtained with other non-morphologic techniques such as flow cytometry and genetic analysis, and above all the clinical findings. The two approaches effectively complement each other. Cases of AML associated with marrow fibrosis represent a diagnostic challenge. Among the fibrotic AML, two subtypes which typically cause the greatest diagnostic difficulties are acute megakaryoblastic leukemia (AMKL) and acute panmyelosis with myelofibrosis (APMF; acute myelosclerosis) ${ }^{[14]}$.

\section{Conclusion}

Our study underscores the fact that light microscopic features of peripheral smear and bone marrow still remain mainstay in the diagnosis of acute leukemias. However, with newer modalities of therapy and rewarding curative results in hematological malignancies, the combination of morphology, cytochemistry, immunophenotyping and cytogenetics have become gold standards for arriving at a specific diagnosis.

\section{Conflicts of interest- None}

\section{References}

1. Catovsky D, Tavares de Castro J. The classification of acute leukemia (AL) and its clinical significance. Schweiz Med Wochenschr. 1983; 113(40):1434-7.

2. Vardiman JW, Thiele J, Arber DA, et al. The 2008 revision of the World Health Organization (WHO) classifications of Myeloid Neoplasms and Acute Leukemia: Rationale and Important Changes. Blood. 2009; 114(5):937-51.

3. Schumacher, Harold, et al. Acute leukemia, Clin labmed. 2002; 22: 153-92.

4. Hoffman Ronald, Benz J Edward, Shattil J Sanford, Fury Bruce, et al. Hematology Basic principles and Practice, V ed, Chapter 59, page no 921-31.

5. Robert W. McKenna. Multifaceted Approach to the Diagnosis and Classification of Acute Leukemias Clinical Chemistry Aug 2000, 46 (8) 1252-1259;

6. Vardiman JW, Harris NL, Brunning RD. The World Health Organization Classification of the Myeloid Neoplasms. Blood. 2002; 100: 2292-302.

7. D'Costa G, Siddiqui HM, Pradhan RM, et al. Pattern of leukemias: a ten-year incidence study of 242 cases. J Postgrad Med. 1989; 35(4): 191-5.

8. Kusum A, Negi G, Gaur DS, et al. Hematological malignancies diagnosed by bone marrow examination in a tertiary hospital at Uttarakhand, India. Indian J Hematol Blood Transfus. 2008;24(1):7-11.

9. Advani $\mathrm{SH}$, et al. A study of 1126 leukemia cases, epidemiologic and end result analysis: Ind J Cancer. 1979;16:817. 
10. Shome DK, et al. The leukemias at presentation: Clinical, Demographic and cytologic variables: Ind J Cancer. 1985; 194-209.

11. Mathur SK, et al. Clinical profile of acute leukemias: A study of 50 cases: Indian Practitioner. 1993;46:171-74.

12. Bain BJ. Auer rods or Mc Crae rods. Am J of Hematol. 2011; 86(8): 689.

13. Loffler H, Gassmann W. Morphology and cytochemistry of acute lymphoblastic leukemia. Baillieres Clin Haematol. 1994; 7(2): 263-72.

14. Atillio Orazi. Histopathology in the Diagnosis and Classification of Acute Myeloid Leukemia, Myelodysplastic Syndromes, and Myelodysplastic/ Myeloproliferative Diseases. Pathobiology 2007;74:97-114. 